Conclusions Low healthcare affordability is associated with lack of high-quality and timely surgery especially among NHB and Hispanic patients, indicating the need for interventions promoting equitable access to guideline-adherent care for all OC patients.

\section{EPV251/\#612 ASSOCIATIONS OF HEALTHCARE AFFORDABILITY WITH UTILIZATION OF SUPPORTIVE CARE MEDICATION AMONG WHITE, BLACK, AND HISPANIC OVARIAN CANCER PATIENTS}

1,20 Ohamadike*, ${ }^{2} \mathrm{~L}$ Wilson, ${ }^{1} \mathrm{~S}$ Luke, ${ }^{2} \mathrm{~T}$ Akinyemiju. ${ }^{1}$ Duke University School of Medicine, Department of Obstetrics and Gynecology, Durham, USA; ${ }^{2}$ Duke University School of Medicine, Department of Population Health Sciences, Durham, USA

\subsection{6/ijgc-2021-IGCS.322}

Objectives Black cancer survivors report higher rates of depression, pain, and fatigue compared to other races/ethnicities. We sought to evaluate the association between healthcare affordability (HA) and supportive care (SC) medication utilization among ovarian cancer (OC) patients by race.

Methods Data for Non-Hispanic White (NHW), Non-Hispanic Black (NHB), and Hispanic OC patients diagnosed in 20082015 in the SEER-Medicare database was analyzed. Factor analysis was used to determine a composite score for HA. SC medication utilization included receipt of antidepressants, psychostimulants, and analgesics. Multivariable log-binomial regression was used to evaluate associations between race/ethnicity, affordability, and SC medication use in the 6 months following OC diagnosis with adjustment for patient clinical characteristics. Sub-group analyses were performed evaluating these associations among late-stage (stage III-IV) patients.

Results The cohort included 3,697 patients: 86\% NHW, 6\% NHB, and $8 \%$ Hispanic. In adjusted models, patients with lower affordability scores were less likely to receive antidepressants compared to those with higher affordability scores (all stage: aOR $0.84 ; 95 \%$ CI $0.73-0.96$ and late-stage: aOR 0.85 ; 95\% CI 0.72-0.99). Additionally, NHB were less likely to receive antidepressants compared to NHW patients (all stage: aOR 0.46 ; $95 \%$ CI $0.33-0.63$ and late-stage: aOR 0.36 ; 95\% CI $0.24-0.56)$. There was no association between affordability and psychostimulant or analgesic utilization.

Conclusions Low healthcare affordability is associated with lower utilization of antidepressants among OC patients, and NHB patients are less likely to receive antidepressants. This indicates the need for interventions targeting more affordable and equitable access to these supportive care medications.

\section{EPV252/\#67 SENTINEL NODE MAPPING IN ENDOMETRIAL CANCER USING HYSTEROSCOPIC INJECTION OF INDOCYANINE GREEN AND NEAR-INFRARED FLUORESCENCE IMAGING}

${ }^{1} \mathrm{G}$ Bogani ${ }^{*},{ }^{2} \mathrm{~A}$ Ditto, ${ }^{1} \mathrm{~F}$ Raspagliesi. ${ }^{1}$ Fondazione IRCCS Istituto Nazionale dei Tumori di Milano, Gynecologic Oncology, Milano, Italy; ${ }^{2}$ Fondazione IRCSS Istituto Tumori di Milano, Gynecologic Oncology, Milano, Italy

10.1136/ijgc-2021-IGCS.323
Objectives To report on the performance of hysteroscopic injection of indocyanine green (ICG) for sentinel lymph node mapping (SNM) in endometrial cancer

Methods This is a retrospective cohort study of consecutive endometrial cancer patients who had SNM via hysteroscopic injection of IGC between 2013 and 2017. Detection rate, accuracy, and oncologic outcomes were evaluated

Results Charts of 52 patients were evaluated. At least one sentinel node was detected in $95 \%$ of patients. Bilateral pelvic mapping was found in $74 \%$ of cases. In $45 \%$ of cases, SLNs mapped in both pelvic and para-aortic nodes, and four cases $(8 \%)$ in the para-aortic area, only. In three patients $(6 \%)$ sentinel nodes were found in aberrant (parametrial/presacral) areas. Seven $(13.5 \%)$ patients were diagnosed with nodal involvement. Low volume disease was observed in four $(8 \%)$ patients ( 2 with isolated tumor cells and 2 with micrometastasis). After a median (range) follow-up of 34.7 (10, 61) months, five (9.6\%) patients developed recurrences: two abdominal/distant, one vaginal, and one nodal (in the paraaortic area in a patient diagnosed with endometrioid G1 endometrial cancer and isolated tumor cells in a pelvic node). No patient died of disease.

Conclusions Hysteroscopic injection of ICG ensures delineation of lymphatic drainage from the tumor area, thus achieving accurate detection of sentinel nodes. Further evidence is warranted to assess the role of hysteroscopic injection in identifying extrapelvic sentinel nodes.

\section{EPV253/\#125 TWO CASES OF OBTURATOR NERVE COMPLETE TRANSECTION DURING LAPAROSCOPIC PELVIC LYMPH NODE DISSECTION FOR ENDOMETRIAL CANCER}

${ }^{1} \mathrm{~T}$ Mogami* ${ }^{1}$ Y Shinbori, ${ }^{1} \mathrm{~F}$ Yamamoto, ${ }^{1} \mathrm{~S}$ Iwaizumi, ${ }^{1} \mathrm{~T}$ Tachibana, ${ }^{1} \mathrm{~S}$ Nakagawa, ${ }^{1} \mathrm{E}$ Onuma, ${ }^{1} \mathrm{~S}$ Saito, ${ }^{1} \mathrm{R}$ Asano, ${ }^{2} \mathrm{E}$ Miyagi, ${ }^{1} \mathrm{H}$ Sakakibara. 'Yokohama City University Medical Center, Gynecology, Yokohama, Japan; ${ }^{2}$ Yokohama City University, Obstetrics and Gynecology, Yokohama, Japan

\subsection{6/ijgc-2021-IGCS.324}

Objectives For early-stage endometrial cancer, laparoscopic surgery is well established in many countries. In Japan, the procedure is covered by insurance from 2014. Since then, laparoscopic surgeries for gynecological cancers have been performed by not only gynecolo-oncologists but also laparoscopic qualified gynecologists. To review the safety of our cases, this IRB-approved study was performed.

Methods Operative cases of endometrial cancer were reviewed retrospectively.

Results Out of 94 stage I endometrial cancer cases who underwent laparoscopic surgery, total laparoscopic modified radical hysterectomy + laparoscopic pelvic lymphadenectomy was performed for 22 patients. Median operative duration was 238 minutes, and median blood loss was $100 \mathrm{~mL}$. We experienced two cases of obturator nerve complete transection. Both of the surgeons were laparoscopic board certificated. In both cases, right obturator nerve was cut near to the branch of internal iliac vein. Surgical video can be revisited for one patient. The nerve covered with lymphatic tissue was dragged out medially under the internal iliac branch, and cut 